\title{
Face à l'avortement : exigences éthiques et dilemme moral à Ouagadougou (Burkina Faso)
}

\section{Ramatou Ouedraogo}

\section{OpenEdition}

\section{Journals}

Édition électronique

URL : http://journals.openedition.org/anthropodev/306

DOI : 10.4000/anthropodev.306

ISSN : 2553-1719

Éditeur

APAD - Association pour l'anthropologie du changement social et du développement

Édition imprimée

Date de publication : 1 septembre 2014

Pagination : 123-141

ISBN : 9791093476018

ISSN : 2276-2019

Référence électronique

Ramatou Ouedraogo, «Face à l'avortement : exigences éthiques et dilemme moral à Ouagadougou

(Burkina Faso) », Anthropologie \& développement [En ligne], 40-41 | 2014, mis en ligne le 01 septembre

2016, consulté le 19 avril 2019. URL : http://journals.openedition.org/anthropodev/306 ; DOI :

10.4000/anthropodev.306

La revue Anthropologie \& développement est mise à disposition selon les termes de la Licence Creative Commons Attribution 4.0 International. 


\title{
Face à l'avortement : exigences éthiques et dilemme moral à Ouagadougou (Burkina Faso)
}

\author{
Ramatou Ouedraogo
}

Au Burkina Faso, l'avortement provoqué fait l'objet d'une restriction juridique et d'une réprobation sociale. Cette situation contribue à en faire un sujet sensible aussi bien pour les acteurs en présence que pour le chercheur qui a pris pour objet cette pratique. Enquêter sur les terrains sensibles, notamment sur les objets illicites et secrets, nécessite de la part du chercheur d'adopter des postures dont les clés ne sont pas toujours offertes. L'une des postures est le rapprochement d'avec son objet et la création de liens fondés sur la confiance. Cette posture permet de rendre visible l'objet, de le laver de sa tension et de favoriser la parole autour. Cependant, elle met à mal le souci de neutralité souvent cher à la relation d'enquête. Le présent article rend compte d'expériences de malaise et de prises de risque du chercheur en situation d'enquête sur l'avortement provoqué au Burkina Faso. L'enquête s'articule autour de 18 mois passés auprès de femmes ayant vécu des expériences d'avortement et/ou en quête d'avortement. Le désir des femmes en quête d'avortement d'impliquer le chercheur dans leur démarche (les aider à trouver un avorteur, donner un avis sur la décision d'avorter, sur le type de méthode que propose autrui, etc.) et la confrontation à la détresse entourant cette demande sont des formes d'implication qui se posent aujourd'hui au chercheur sur son terrain. Ces sollicitations ont des implications morales, éthiques et aussi juridiques intenses pour le chercheur. Réagir à ces sollicitations (répondre aux attentes de la femme ou non) signifie d'une manière ou d'une autre une forme d'engagement susceptible de plonger le chercheur dans une souffrance (au regard de ses valeurs propres, du risque d'enfreindre une loi versus de rester inactif face à la détresse d'autrui, etc.) dont la prise en compte s'avère cruciale pour repenser les rapports à l'objet d'étude dans la recherche.

In Burkina Faso, induced abortion is subject to legal restriction and social stigma. This situation makes it a sensitive issue both for the actors involved and the researcher studying abortion. Conducting research on sensitive issues, especially when these are illicit and secret, requires the researcher to adopt stances which are not always 
predictable. One of these stances involves establishing close relationships based on trust with the respondents. This allows the researcher to render visible invisible objects, diminish tensions and promote dialogue. However, such a stance puts in peril the impartiality of the researcher, which is often crucial to the scientific process. This article is an account of experiences of unease and risks taken by researcher of induced abortion in Burkina Faso. It focuses on 18 months of fieldwork on women who have experienced abortion and/or in the quest for abortion. The desires of women in their quest for abortion to involve the research in their mission (help them to find an abortionist, advice them to (or not to) abort, advice them on preferred methods, etc.) and dealing with the distress concerning these demands, are some of the forms of involvement that the researcher is confronted with during fieldwork. These solicitations have intense moral, ethical and legal implications for the researcher. Reacting to these solicitations by responding (or not) to the expectations of women indicates a form of engagement susceptible to immerse the researcher in a distress arising from a conflict with personal values and a risk of breaking the law versus remaining inactive when confronted with the distress of others. The apprehension of this involvement is crucial to the rethinking of the relations of the researcher to the respondents in the research process.

\section{Introduction}

La question de la juste distance entre le chercheur et son objet de recherche a toujours été un sujet important pour les sciences sociales. La situation d'enquête socio-anthropologique exige en théorie du chercheur une neutralité dans ses rapports à son objet d'étude (Weber, 1959). Dans la pratique cependant, il est de plus en plus ressorti que cette neutralité était quasi impossible pour le chercheur, et qu'elle relève plus d'une définition normative de ce que devrait être une science que d'une description objective de ce qu'elle est (Fassin, 1999). Norbert Elias (1995: 10) explique, en effet, que la question de l'engagement et de la distanciation, loin d'être l'apanage de la recherche, est inhérente aux interactions du quotidien entre les individus. Ces derniers seraient animés d'impulsions qui oscillent entre le désir d'engagement et celui de la distanciation, et qui déterminent le cours de leurs actions. Le chercheur en tant qu'acteur d'une société donnée ou parfois de celle qu'il prend comme objet d'étude 
(Bila, 2009; Ouattara, 2004) s'inscrit, de fait, dans ces impulsions d'engagement et de distanciation, et est ainsi invité à penser ensemble cette dualité dans sa pratique. L'idéal de la "neutralité axiologique » n'étant pas atteignable, la théorie éliasienne permettrait, selon Didier Fassin (1999), de penser la tension entre l'engagement et la distanciation, en la représentant sous la forme d'un gradient plutôt que comme deux entités séparées.

Cependant, comme il le relève à juste titre, il existe une diversité des formes d'engagement et de distanciation :

"Si la conceptualisation que propose Norbert Elias permet donc de mieux rendre compte de la réalité que ne le faisaient notamment les approches durkheimienne et weberienne, elle exprime en revanche mal la diversité des formes d'engagement et de distanciation correspondant à la multiplicité des paradigmes des sciences sociales. » (Fassin, $1999: 4$ )

Ainsi, d'un terrain à l'autre, d'un sujet à un autre, engagement et distanciation n'ont pas le même sens. En fonction des objets de recherche, s'engager peut prendre plusieurs formes et avoir diverses implications. Je me propose de poursuivre le débat sur la diversité des formes d'engagement du chercheur et de ses implications, notamment les engagements à risque (Pirinoli, 2004) susceptibles de générer un inconfort ethnographique (Agier et al., 1997), voire une souffrance, chez le chercheur. L'objectif du présent article est de rendre compte d'expériences de malaise et de prises de risque du chercheur en situation d'enquête sur l'avortement provoqué au Burkina Faso ${ }^{1}$. Il s'articule autour de 18 mois d'enquêtes (entre juillet 2011 et janvier 2013) auprès de femmes ayant vécu des expériences d'avortement et/ou en quête d'avortement. En premier lieu, j'expliquerai en quoi enquêter sur l'avortement au Burkina Faso constitue un terrain "sensible ». Ensuite, je décrirai comment la relation d'enquête sur les sujets sensibles peut contribuer à transformer le chercheur en termes de valeurs et de postures. Enfin, dans le dernier point, je

\footnotetext{
${ }^{1}$ Cette recherche compte pour ma thèse de doctorat en anthropologie, et s'inscrit dans un projet de recherche financé par le Conseil Norvégien de la Recherche.
} 
discuterai des formes que peuvent revêtir les demandes d' " aide " sur ce type de terrain ainsi que leurs implications.

\section{Enquêter sur l'avortement au Burkina Faso : un terrain sensible?}

L'avortement, en tant que " mauvais objet " (Boltanski, 2004) est entouré de difficultés pour le saisir comme objet, de difficultés d'accès au terrain, et pose à l'ethnographe un ensemble de questionnements éthiques. Tout chercheur qui le prend pour sujet est, a priori, susceptible de se confronter à ces difficultés, qu'il choisisse d'enquêter en France, en Tunisie, en Inde ou au Burkina Faso. Toutefois, le statut juridique et social accordé à l'avortement dans ces espaces contribue à différencier les difficultés d'un terrain à un autre. Les contraintes méthodologiques de l'étude de l'avortement dans les contextes de restriction/interdiction juridique et de forte stigmatisation ont été démontrées (Guillaume, 2004), et cela est une des raisons de l'absence d'ethnographie sur la question. II se pose non seulement des difficultés pour estimer la prévalence en raison d'une sousdéclaration des cas d'avortement, mais aussi pour faire évoquer les expériences sur la question car très souvent ces avortements ne sont pas admis par les personnes qui sont directement impliquées (la femme, l'homme, les agents de santé, etc.).

Ainsi, enquêter sur l'avortement au Burkina Faso constitue pleinement un « terrain sensible » (Bouillon, Fresia, Tallio, 2005 : 14) car portant :

"[...] sur des pratiques illégales ou informelles, des individus faisant l'objet d'une forte stigmatisation et sur des situations marquées par la violence, le danger, la souffrance. » (ibid., 2005 : 14)

En effet, le code pénal du Burkina Faso punit l'avortement provoqué en ses articles 383-386 et 388-390. L'article 383 par exemple stipule :

"Est puni d'un emprisonnement d'un à cinq ans et d'une amende de 300.000 à 1.500 .000 francs quiconque par aliments, breuvage, médicaments, manœuvres, violences ou par tout autre moyen, procure ou tente de procurer l'avortement d'une femme enceinte ou suppo- 
sée enceinte qu'elle y ait consenti ou non. Si la mort en résulte, la peine est un emprisonnement de dix à vingt ans. La juridiction saisie peut en outre prononcer l'interdiction professionnelle et/ou l'interdiction de séjour pour une durée qui ne peut excéder cinq ans. " (code pénal, article 383)

L'avortement y est autorisé seulement sur indication médicale (grossesse présentant un risque pour la mère ou malformation du fœtus) ou juridique (grossesse résultant d'un viol ou d'un inceste), selon l'article 387.

Parallèlement à cette restriction juridique, l'avortement provoqué fait l'objet d'une forte réprobation sociale comme religieuse. II s'apparente à un crime, à une déviance morale qui est socialement et religieusement reprouvée. Selon une enquête menée en 2010 par le Centre pour la Gouvernance Démocratique sur les Burkinabè et leurs valeurs, l'avortement serait la troisième pratique jugée inadmissible après l'homosexualité et la prostitution. L'avortement est par conséquent une pratique illégale (à l'exception de ceux autorisés par la loi) et clandestine, peu dite mais connue de tous, et la forte réprobation induit une stigmatisation vis-à-vis des femmes rendues coupables de cette pratique. Cette situation contribue à en faire un sujet sensible aussi bien pour les acteurs en présence (les femmes, leurs proches, les agents de santé, les décideurs), que pour le chercheur qui a pris pour objet cette pratique. Le champ de l'avortement au Burkina Faso est un lieu de confrontation de convictions diverses où les acteurs naviguent entre non-dits, faux semblants, censures et suspicions. Enquêter dans ces conditions comporte plusieurs contraintes liées notamment à la relation d'enquête que construit l'ethnographe avec ses " enquêtés " (Bouillon, Fresia, Tallio, 2005). En somme, comment enquêter sur ce qui peut être considéré comme une douleur pour les uns et une déviance pour les autres ? Comment faire une recherche sur un objet connu de tous mais invisible? Comment investiguer sur une pratique faisant l'objet, comme le dit Luc Boltanski (2004), d'une " mauvaise foi sociale »?

Un des moyens pour contourner la difficulté d'accès au terrain a été pour moi de passer par la porte des soins après avortement, qui me permettait d'observer non seulement les pratiques entre soignants et soignées, mais aussi de parer à la difficulté d'identification des femmes. II a fallu au quotidien travailler à ménager les susceptibilités, surveiller mes 
propos, parfois faire preuve d'autocensure au risque de me faire cataloguer très rapidement de " pro-avortement". La question "que comptez vous proposer à la fin de cette étude? J'espère que ce n'est pas pour proposer qu'on légalise l'avortement " m'a souvent été posée, me rappelant ainsi régulièrement la méfiance des enquêtés et la réprobation qui entoure la pratique de l'avortement. Il est difficile dans un tel contexte de se positionner, entre d'une part des soignants et des acteurs politiques généralement réprobateurs, et d'autre part des patientes vulnérables en quête d'attention et de compassion, sans tomber dans le parti pris.

\section{De l'illusion d'une possible neutralité à l'implication : quand la relation d'enquête participe à la transformation de soi}

Lorsque j'ai entamé cette enquête sur l'avortement, j'avais mes convictions personnelles sur cette pratique, en lien avec mes croyances religieuses et mon éducation. II me revient en mémoire ces propos entendus plusieurs fois lors de séances de prêche :

"Lorsqu'une femme qui a fait un avortement meurt, le jour du jugement dernier elle retrouve l'enfant dont elle a ôté la vie qui l'attend les mains fermées, et qui les ouvrent à son arrivée pour lui remettre son châtiment, l'enfer. »

En effet, j'ai été éduquée dans l'idée que les femmes qui avaient recours à l'avortement étaient des femmes de "mauvaise vie ". En conséquence, je m'étais promis, en tant que jeune femme issue d'une famille musulmane, de ne jamais avoir recours à cette pratique et je voyais d'un mauvais œil les jeunes femmes qui étaient soupçonnées d'avoir avorté. Je me suis ainsi engagée sur ce terrain avec des convictions contre l'avortement, et le fait de baigner dans un univers me confortant dans cette position a créé en moi l'illusion d'une possible distanciation, position exacerbant le sentiment d'altérité. Je me disais : "Elles, ce sont celles qui sont en infraction au regard des normes et avec qui je n'ai rien à voir, donc je les traiterai comme des choses ». Et une fois l'évidence de cette possible distanciation acquise, mon non-engagement allait de soi, puisqu'il faut se sentir proche d'une cause pour nourrir le désir de s'impliquer (défendre leur cause par exemple). 
Le défi pour moi se situait à un autre niveau : comment faire pour ne pas laisser mes perceptions de l'avortement influencer mes rapports à mes informateurs et à mes données car :

"Certes, nul ne saurait empêcher le savant d'avoir des "convictions personnelles", pourvu toutefois qu'il ne les mêle pas à ses recherches et qu'il ne les enseigne pas du haut de sa chaire. " (Fassin, 1999 : 44)

Il m'incombait alors d'œuvrer à ce que mes convictions personnelles n'entachent pas mes rapports à ces jeunes femmes. Cependant, au fil des rencontres, je me suis aperçue, comme le montrait Didier Fassin, que "I'homme ne se désengage jamais complètement du monde, quel que soit le degré de désenchantement qu'il produise à son égard » (1999: 44).

Le caractère sensible d'un sujet comme celui de l'avortement ainsi que la personnalité des enquêtés et de l'enquêteur obligent à développer parfois des astuces afin de réussir la "négociation invisible " (Olivier de Sardan, 1995), au risque pour le chercheur d'échouer dans sa mission. En effet, le rôle d'informateur - qui est d'ailleurs difficile à endosser - sur ce type de sujets suscite crainte, atermoiements, suspicions et mutisme. Mon statut d'enquêtrice burkinabè vivant dans la même ville que les enquêtées, mon assimilation à un membre du personnel des structures de santé ou les doutes quant à mon statut véritable ne favorisaient pas la discussion sur un sujet qui relevait du domaine du secret pour la plupart de ces femmes. Elles étaient généralement toutes méfiantes au premier contact : " $n$ 'estelle pas une espionne à la solde de la police ?" "Ne serais-je pas dénoncée ? "Ainsi, entre fausses adresses, faux contacts et faux rendez-vous, entretiens expéditifs et prétextes douteux, j'ai été confrontée à une résistance pesante, parfois décourageante. Néanmoins, ces situations qui n'ont rien de fortuit illustrent ce que Estelle d'Halluin (2005) qualifie d'« énigme réciproque " pour le chercheur et pour les informateurs. Cette énigme réciproque, loin de constituer un échec (ou d'être ressentie comme tel) constitue en fait le signe d'une ébauche de la relation d'enquête entre le chercheur et l'informateur (Losonczy, 2002). Face à ce type de terrains "sensibles", les formes d'adaptation mises en œuvre par les chercheurs sont légion. Sophie Divay (2004) en France, par exemple, a opté pour une 
ethnographie à l'insu de ses informatrices, avec tous les enjeux déontologiques et méthodologiques que cela implique. Elle a choisi, en effet, d'utiliser les données recueillies au cours d'entretiens préalables à des demandes d'IVG dans le cadre de son activité en tant que conseillère conjugale dans une association.

Pour ma part, la négociation invisible passait par la posture empathique. J'ai réalisé que mon statut de jeune femme célibataire apparaissait a priori comme un élément susceptible de nous rapprocher dans la mesure où certaines expériences nous étaient communes à quelques exceptions près (la complexité des relations amoureuses par exemple). J'ai donc pris le parti d'exploiter ces atouts afin de me rapprocher d'elles, c'est-à-dire privilégier la "posture empathique " pour les mettre en confiance sur des discussions autour d'évènements intimes et souvent douloureux. Je suis parvenue au fil des rencontres à faire disparaître cette méfiance et à instaurer une certaine confiance qui puisse les amener à se dire. Comme dans toute interaction, des liens interpersonnels ont fini par se créer avec le temps, et de l'inconnue potentiellement dangereuse, je suis devenue la "tantie ", la grande sœur, la confidente qu'on appelle désormais pour demander conseil, se confier, ou solliciter un soutien sans risque que l'information ne soit divulguée dans leurs réseaux respectifs. En d'autres mots, on pourrait dire que j'étais "l'amie inconnue ".

Il ne s'agissait pas pour elles de m'assimiler comme membre de leur réseau à part entière, mais plutôt de m'y intégrer avec mes spécificités, à savoir comme une "intellectuelle", inconnue de leur réseau social, avec possibilités de répondre à certaines de leurs préoccupations, etc. En retour, le temps que je leur accordais me permettait de les côtoyer régulièrement - partager leurs craintes et angoisses, leurs projets et aspirations, leurs difficultés, leurs rapports avec les gens autour d'elles -, de dresser leur parcours et de découvrir les motivations profondes de leur " geste ". Le fait de privilégier des relations personnalisées pour instaurer la confiance et lever la barrière d'accès aux données a transformé à mon insu ma vision de l'avortement. De celle qui juge sans le dire, je suis passée à celle qui comprend, qui se dit qu'elle n'aurait peut-être pas fait mieux et adhère à une cause. Ce nouveau statut (sur le plan des perceptions de l'avortement) et cette adhésion à la cause impliquaient pour moi de les 
aider à surmonter "leur souffrance " à laquelle j'étais devenue désormais sensible. J'ai ainsi découvert qu'enquêter sur des expériences d'avortement peut faire passer le chercheur d'une perception à une autre de l'avortement, le rendre par moment partisan, ou même faire naître chez lui la tentation de se porter défenseur des "causes perdues". A partir de ce moment, le défi devenait tout autre : comment faire pour ne pas dépasser la " ligne rouge " surtout lorsque le terrain vous sollicite régulièrement et veut que vous soyez actif?

\section{Ethnographie de formes de sollicitation à l'endroit du chercheur}

Ma relation avec Maria et Afia, deux jeunes femmes qui ont connu des expériences d'avortement, permet d'illustrer le type de sollicitations auxquelles j'ai pu être confrontée.

Maria est une jeune femme de 23 ans dont j'ai fait la connaissance en septembre 2011 au cours de sa prise en charge à la suite d'une complication après une interruption volontaire de grossesse (IVG) clandestine. Elle avait beaucoup hésité avant d'accepter ma proposition de la rencontrer pour un entretien. C'est après plusieurs relances par téléphone qu'elle avait fini par me fixer un rendez-vous chez elle, trois mois après sa prise en charge. Quand je m'y suis rendue, j'ai retrouvé une jeune femme nerveuse qui a fini par m'avouer :

"Toute la nuit je n'ai pas dormi, je me posais des questions, et si c'est la police qui l'envoie? Entre temps j'ai même failli éteindre mon téléphone. »

Aussi, pour dissiper ses craintes et son malaise, je me suis contentée d'une discussion informelle qui n'a rien eu à voir avec son avortement. Par la suite, en multipliant les échanges au téléphone et les visites chez elle, nous avons sympathisé et sommes parvenues à avoir notre premier vrai entretien. J'ai ainsi appris qu'elle était née dans un village à environ $300 \mathrm{~km}$ de Ouagadougou et qu'elle est venue dans la capitale sous le couvert d'un « oncle ». Maltraitée par ce dernier, elle avait décidé de louer un petit studio et avait trouvé un emploi de serveuse dans un " glacier " pour 
subvenir à ses besoins. Cependant, au chômage depuis deux mois, elle éprouvait des difficultés à payer son loyer et le propriétaire la menaçait d'expulsion. J'ai aussi découvert que l'homme qui " l'avait poussée à avorter " avait mis fin à leur relation amoureuse deux semaines après sa sortie de l'hôpital. Cet abandon et la précarité dans laquelle elle vivait lui avaient donné plusieurs fois des envies de suicide. Elle en avait, d'ailleurs, profité pour me signifier tout le bien que lui faisaient nos échanges et rencontres :

"Souvent tu es laissé à toi-même, et tu fais des choses que tu regrettes. Depuis que je te connais je me sens moins seule, tu me donnes des conseils de grande sœur, si je t'avais connue depuis, il y a beaucoup de choses que je n'aurai pas fait. »

A la fin de cet entretien, Maria m'a appris qu'elle se sentait « bizarre " depuis quelques jours :

"Hier j'ai fait du tô et j'ai mangé, mais je n'arrivais pas à digérer, mon ventre était gonflé et puis ça revenait vers ma poitrine, donc je suis allée boire du thé avec du citron pour essayer de faire passer ça, mais ça va pas. Depuis ce matin je sens que la nourriture là est ici (elle montre sa gorge). Est-ce que tu vois ? Donc j'ai tout fait pour vomir ça juste avant que vous ne m'appeliez. Mais j'ai toujours l'impression que c'est toujours là. Je ne sais pas ce qui m'arrive. "

Tout en la rassurant, je lui ai proposé de faire un test de grossesse, ce qui a eu pour effet de la troubler :

"Je ne peux pas être enceinte, mes seins ne sont pas gonflés, donc ça peut pas être ça. Si c'est ça walaï la mort est mieux. »

Devant mon insistance, elle a fini par accepter l'idée du test à la condition que je l'assiste au moment de la réalisation. J'espérais au fond de moi qu'elle n'était pas enceinte car je m'étais prise de sympathie pour elle et m'imaginais la difficulté dans laquelle une éventuelle grossesse allait la placer. Le lendemain matin, le test s'est malheureusement révélé positif et je me suis retrouvée avec une jeune femme en pleurs à consoler pendant toute une matinée. 
Elle m'a alors raconté les conditions dans lesquelles la grossesse avait été contractée :

"Quand mon copain m'a quittée j'étais triste, il y avait l'avortement aussi que je n'arrivais pas à oublier, donc j'ai décidé de m'amuser pour oublier. J'ai une amie ivoirienne qui m'a fait connaître un de ses frères ivoiriens [...]. Chaque fois le gars m'appelait au téléphone et venait me voir, je me suis dit pourquoi pas, au moins il va m'aider à oublier les choses, je n'espère rien, comme je ne prends plus un gars au sérieux, c'est juste pour m'amuser? Quand j'ai commencé à sortir avec lui, il m'a dit que moi je suis le genre de filles que eux ils aiment avoir à côté d'eux parce que je suis simple. Tous ses amis le connaissaient dans son affaire de femmes, mais bizarrement quand il m'a connue là, il ne sortait avec aucune fille, et ses amis même étaient surpris de nous voir ensemble aussi longtemps [...]. Donc j'ai commencé à le prendre au sérieux. [...] II est reparti en Côte d'lvoire depuis deux semaines, il $m^{\prime} a$ dit que s'il arrive il va m'appeler et que s'il revient à Ouaga il va m'épouser. Mais depuis qu'il est parti il n'a jamais pris même son téléphone pour m'appeler. Pourtant quand il était là il disait que lui il veut un enfant. »

La décision a été prise sur le coup d'informer le compagnon en question. Elle apprendra alors qu'il est marié avant qu'il ne lui raccroche au nez. Pendant une semaine Maria a hésité entre garder sa grossesse et avorter : mener la grossesse à son terme risquait d'accentuer la précarité dans laquelle elle vivait (enceinte sans domicile à cause des impayés de loyers, obligation d'assumer seule la grossesse, réduction de ses chances qu'un autre homme lui prête attention, etc.); parallèlement elle avait peur de revivre des douleurs similaires à celles du précédent avortement et d'en mourir. J'ai essayé de l'accompagner durant cette période d'hésitation faite surtout de détresse et de larmes. Elle aboutira à la conclusion suivante :

"Je sais que je ne peux pas garder cette grossesse, mais si c'est pour revivre ce qui s'est passé la dernière fois, je préfère garder et laisser Dieu faire, s'il faut je vais sortir pour mendier pour qu'on mange. "

Pour à la fin me demander de l'aider : 
"Grande scur c'est Dieu qui t'a envoyée vers moi pour me sauver, aide-moi s'il te plait!»

La seconde histoire est celle d'une jeune femme qui avait été reçue en consultation en octobre 2012 pour une grossesse non désirée communément appelée (en sigle) GND par les conseillères dans les structures de prise en charge. Ce jour là, la sage-femme responsable de la clinique et une des conseillères m'ont sollicitée pour les aider à gérer le " cas » :

"Ça fait presque deux heures qu'on lui parle, mais elle ne fait que pleurer seulement, qu'elle a été violée et elle ne peut pas garder la grossesse. Viens nous aider, peut être que tu pourras la calmer." (propos de la conseillère)

C'est ainsi que je me suis retrouvée devant Afia, une étudiante de 20 ans en quête d'avortement au motif de viol. Elle serait allée dans un " maquis " pour oublier des soucis financiers et aurait perdu connaissance après qu'un homme lui ait proposé un verre. Après cet épisode, elle aurait constaté l'absence de ses règles, elle serait allée en consultation, et finalement des examens (un test de grossesse et une échographie) avaient révélé une grossesse de cinq semaines. Elle est ainsi venue dans cette structure pour bénéficier d'un avortement sécurisé conformément aux dispositions de la loi, mais elle ne disposait d'aucune preuve du viol.

Après avoir relaté à mon tour les faits à la sage-femme qui était responsable de la structure, nous avions décidé de la transférer vers le service de maternité du centre hospitalier universitaire. Mais avant cela, elle devait refaire une échographie pour permettre à la sage-femme de juger de la viabilité de la grossesse :

"De toute façon, comme on a dit qu'il faut vérifier dans une semaine, peut-être même que la grossesse n'est pas bonne, peut être que l'œuf n'est pas bon, donc patiente, dans une semaine on va refaire l'échographie pour voir. Même si tu n'as pas l'argent il faut venir on va t'aider à faire l'échographie. " (propos de la sage-femme)

Afia s'est remise à pleurer en entendant ces propos et m'a dit après le départ de la sage-femme : 
"Elles ne veulent pas m'aider. Et si dans une semaine elles me disent qu'elles ne peuvent rien faire ? Je ne peux pas partir d'ici, il faut qu'on m'aide. »

A force de persuasions, je suis arrivée à lui faire accepter l'idée d'attendre une semaine avant toute décision. Et je me suis engagée à l'épauler durant cette période d'attente (je lui ai donné mon numéro de téléphone et mon adresse). Cependant, après son départ les sages-femmes avaient mis en doute la véracité du viol et ont conclu qu'elle mentait. Elles auraient constaté l'absence de concordance entre la date du viol et l'âge de la grossesse. Bien plus, une autre sage-femme disait l'avoir reçue au cours de sa garde et elle avait prétexté un inceste. Partant de ce doute et de mon souci de neutralité, j'ai décidé alors de ne plus la revoir et de renoncer à son " cas ». Une semaine après, l'échographie a révélé une grossesse évolutive. Revenue à la clinique pour présenter les résultats, aucune des sages-femmes n'avait voulu la recevoir, et j'avais fait de même car je ne me sentais pas capable de faire face à sa détresse. Je craignais également les interprétations que pouvaient susciter une trop grande attention de ma part. Je me suis donc défilée avec la conscience lourde. En fin de journée ce jour-là, lorsque j'ai rejoint les locaux de l'institution où j'étais accueillie pour ma thèse, j'ai trouvé à l'accueil une note de Afia à mon intention:

"Bonjour tantie Ramatou, je suis passée à $X$ mais les dames-là ne veulent pas m'aider. Appelle-moi stp, je veux te dire la solution que j'ai trouvée, je suis dans le désespoir total, si tu m'abandonnes je n'aurai plus de choix. Tu es mon seul espoir avant que je ne commette l'irréparable. Afia, mon téléphone est le ...»

Que faire face à un tel message ?

\section{De l'aide valorisée à la complicité. La relation d'enquête, entre exigences éthiques et dilemme moral}

Comme le soulignait Blandine Bila, la relation d'enquête met en contact :

"[...] d'une part des chercheurs en quête d'informations et prêts à s'investir méthodologiquement, éthiquement et matériellement, 
d'autre part des participants démunis, isolés pour la plupart, et en quête de soutien thérapeutique, financier, relationnel et, bien souvent, le tout à la fois. » (Bila, 2009 : 5)

Le chercheur peut ainsi se retrouver dans une situation de don et de contre-don dans ses rapports aux enquêtés : les enquêtés lui fournissent l'information et en retour s'attendent à une rétribution (matérielle, financière, morale). Florence Bouillon (2005) distingue trois types de contredons dans les attentes que les enquêtés peuvent avoir vis-à-vis du chercheur : il s'agit de "la présence et l'écoute ", de "l'extériorité, marqueurs sociaux et gratification symbolique ", et enfin de "l'aide ».

Dans le cas de mon enquête, les jeunes femmes n'avaient, a priori, pas d'attentes vis-à-vis de moi. Elles étaient plutôt méfiantes à l'idée de se confier sur un sujet autour duquel la discrétion était de mise, car la divulgation de leur secret impliquait pour elles un risque de détérioration de leurs liens sociaux et des poursuites judiciaires. Mais une fois la confiance instaurée, je me suis aperçue que la présence et l'écoute étaient généralement les premières choses dont elles étaient en demande. Les femmes qui ont eu recours à un avortement provoqué sont susceptibles d'être confrontées à un traumatisme post-avortement (Allard et Fropo, 2007) qui nécessite à leur égard une attention et parfois un suivi psychologique. Dans les contextes où l'avortement est autorisé et se pratique officiellement, des mécanismes sont mis en place pour accompagner la femme dans son deuil à travers la possibilité d'un accompagnement psychologique par exemple. Au Burkina Faso par contre, la restriction juridique et le caractère stigmatisant de l'avortement font que cette souffrance est «interdite » puisque très peu de personnes sont disposées à l'écouter. D'ailleurs, l'évoquer reviendrait à ouvrir la voie à des épisodes de stigmatisation et parfois de violence. Une de mes informatrices, une élève de 18 ans, avait été suspendue des activités de son église (protestante) et rejetée par ses parents lorsque les membres de l'église ont découvert qu'elle avait fait un avortement. Les femmes se retrouvent, de fait, contraintes à dissimuler leur souffrance et à gérer quasiment seules le traumatisme postavortement. Lorsque je suis entrée dans la vie de ces femmes, je me suis retrouvée à jouer le rôle de psychologue, de conseillère, de celle qui les accompagne et s'attelle à les aider à dépasser l'épreuve. 
Cependant, l'enquête dans des contextes de forte précarité a tôt fait de rappeler au chercheur que l'écoute et la qualification symbolique ne constituent pas toujours des contre-dons suffisants (Bouillon, 2005 : 85). Ainsi, au fil de la relation, l'écoute comme contre-don peut céder la place à l'aide comme contre-don. Les situations de précarité des enquêtées amènent en effet le chercheur à leur apporter un soutien matériel, c'est-à-dire leur donner de l'argent, les aider à payer des médicaments, leur offrir des présents, mais aussi les accompagner dans certaines démarches telles qu'obtenir un rendez-vous avec un médecin pour elles (parce que la procédure normale s'avère longue), négocier une exonération de frais (échographie, contraception, soins, etc.), les accompagner à des rendez-vous avec les soignants (parce qu'elles pensent que ma présence va leur éviter des situations de stigmatisation), etc. A côté de cette aide, on retrouve aussi les sollicitations auxquelles j'ai été confrontée dans les cas de Afia et de Maria, qui ne sont que deux cas parmi tant d'autres. Le contexte normatif de l'avortement au Burkina Faso transforme la quête de l'avortement, pour bon nombre de femmes, en une sorte de " chemin de croix " fait de souffrance, de solitude, de vulnérabilité, de violence, de danger et de douleurs, dans lequel le réseau relationnel joue un important rôle. Elles tentent généralement leur chance auprès de toutes les personnes susceptibles de les aider dans leur réseau social et parfois en dehors de ce réseau (en allant vers des agents dans les structures sanitaires, vers les vendeurs ambulants de médicaments, vers des tradipraticiens), avec tout de même l'idée qu'elles ne doivent aller que vers ceux qui pourront les aider sans leur nuire socialement. En tant que nouveau membre de leur réseau, je deviens un de leurs recours, et non des moindres. Car comme je le soulignais tantôt je suis intégrée dans ce réseau avec mes spécificités et la demande m'est adressée parce que (1) je suis une femme intellectuelle donc en mesure de les comprendre, (2) elles ont fait ma connaissance dans un univers qui leur laisse penser que je dispose des moyens pour les aider (je collabore avec des soignants, porte la même blouse qu'eux, etc.). Le chercheur que je suis fait place, à ce moment, à l'individu et la demande m'est adressée pour les unes en tant qu'agent de santé ou collaborateur des agents de santé, et pour les autres en tant que " grande sœur », " tantie », ou amie. 
Cependant, aider une personne qui est dans un dénuement matériel telle une malade qui a besoin d'aide pour acheter ses médicaments, des vivres, ou une femme qui a besoin de contraception, ou de soins après un avortement, etc., n'a pas le même sens qu'aider une femme à interrompre sa grossesse. Dans le premier cas il s'agit de ce qu'on peut appeler une aide "gratifiante ", de l'ordre de la charité, qui est prônée et encouragée socialement comme religieusement. Par contre, accompagner une femme dans son désir d'avorter en l'assistant dans sa prise de décision, en lui trouvant un avorteur, ou en donnant son avis sur le type de méthode à utiliser n'a pas la même portée sociale. L'aide dont il est question dans le cas présent, même si elle sort la femme d'une détresse et sauve même sa vie, est une aide non gratifiante au regard des valeurs en présence. Elle peut être source de discrédit et met en danger le statut du chercheur, autant socialement que professionnellement. Car lorsque ce type de soutien est rendu public, il passe du statut " d'aide " à celui de " complicité » et est passible de sanctions (juridiques comme sociales). Le chercheur se retrouve dans ces moments dans un dilemme moral duquel il ne sort jamais indemne. En effet, agir, c'est-à-dire aider la femme à interrompre sa grossesse, implique qu'il va à l'encontre de ses valeurs et des attentes de ses collaborateurs, et ne pas agir lui donne le sentiment d'une "non assistance à personne en danger " vu qu'il dispose des ressources pour l'aider. Afia, par exemple, m'a avoué par la suite qu'elle avait décidé de boire de l'acide pour en finir. Que le chercheur décide dans ces cas de figure d'aider la femme ou non, il sera éprouvé moralement. Cette situation que Michel Agier (1997) nomme "inconfort ethnographique " (et que je nommerai pour ma part "souffrance ethnographique ") comporterait, selon lui, une dimension morale intense qui vient de la demande de compromission individuelle adressée au chercheur par ceux qu'il étudie ou avec qui il coopère.

\section{Conclusion}

Enquêter sur les terrains sensibles, notamment sur les objets illicites et les secrets, nécessite de la part du chercheur d'adopter des postures dont les clés ne sont pas toujours offertes. L'une des postures est le rapprochement d'avec son objet et la création de liens fondés sur la confiance. Cette posture permet de rendre visible l'objet, de le laver de sa tension et de favori- 
ser la parole autour. Cependant, elle met à mal le souci de neutralité souvent cher à la relation d'enquête. La distinction enquêteur/chercheur qui recoupe la traditionnelle réflexion sur les rapports entre distance et proximité (induite par la démarche scientifique) apparaît parfois difficile à tenir durant l'enquête de terrain (Renahy et Sorignet, 2006). Avec l'avènement des terrains sensibles, les chercheurs sont amenés à créer des liens interpersonnels avec leurs " objets ", qui sont ici des personnes dans des situations de vulnérabilité, de souffrance, et/ou parfois très suspicieux. Néanmoins, ces liens interpersonnels confrontent les chercheurs à des sollicitations qui dépassent le cadre de l'enquête et mettent à rude épreuve les tentatives de distanciation du fait que l'objet de la recherche est rendu alors « trop proche » par la relation d'amitié (Bila, $2009: 6)$.

Le désir des femmes en quête d'avortement d'impliquer le chercheur dans leur démarche (les aider à trouver un avorteur, un avis sur une décision d'avorter, sur le type de méthode que propose autrui, etc.) et la confrontation à la détresse entourant cette demande sont des formes d'implication qui se posent aujourd'hui au chercheur sur son terrain. Ces sollicitations ont des implications morales, éthiques et aussi juridiques intenses pour le chercheur. Réagir à ces sollicitations (répondre aux attentes de la femme ou non) signifie d'une manière ou d'une autre une forme d'engagement susceptible de plonger le chercheur dans une souffrance (au regard de ses valeurs propres, du risque d'enfreindre une loi versus de rester inactif face à la détresse d'autrui, etc.). Ce type de terrain sensible rend vulnérable le chercheur (socialement, juridiquement, éthiquement) et celui-ci est constamment mis en demeure de remettre en question ses outils mais aussi ses théories, ses positionnements éthiques, politiques, etc. (Pirinoli, $2004: 181$ ).

Rangées généralement sous le sceau de "facteurs personnels " qu'il est malséant de dévoiler, les relations entretenues par le chercheur sur le terrain apparaissent bien souvent essentielles à la constitution même de l'objet d'enquête, selon Nicolas Renahy et Pierre-Emmanuel Sorignet (2006 : 23). Cependant, selon ces auteurs, reconnaître qu'il entretient des relations affectives ou amicales intenses, c'est-à-dire qu'il appartient dans une certaine mesure aussi à un autre univers, c'est faire courir au chercheur le risque d'être délégitimé par ses pairs ou d'être accusé de man- 
quer d'objectivité. Or, mettre en avant ces soucis de distanciation, c'est prendre le risque de ne pas être accepté par les enquêtés (ou une acceptation factice), de taire certaines informations, de biaiser l'analyse et de mettre à mal, par conséquent, sa mission première qui est d'étudier un phénomène ou un groupe donné.

\section{Bibliographie}

AGIER M. (éd), 1997, Anthropologues en danger : l'engagement sur le terrain, Paris, Jean-Michel Place.

ALLARD F., FROPO J.-R. (2007), Le traumatisme post-avortement, Paris, Salvator.

BILA B., 2009, "Anthropologie "chez soi" auprès de personnes vivant avec le VIH à Ouagadougou: Empathie, méthode et position des acteurs ", Ethnographiques.org, URL : http://www.ethnographiques.org/2008/Bila.

BOLTANSKI L., 2004, La condition fotale: une sociologie de l'engendrement et de l'avortement, Paris, Gallimard.

BOUILLON F., 2005, "Pourquoi accepte-t-on d'être enquêté? Le contre-don au coeur de la relation ethnographique ", in Bouillon F., Fresia M. et Tallio V. (éds.), Terrains sensibles. Expériences actuelles de l'anthropologie, Paris, EHESS : 75-95.

BOUILLON F., FRESIA M., et TALLIO V. (éds), 2005, Terrains sensibles: expériences actuelles de l'anthropologie, Paris, EHESS.

D'HALLUIN E., 2005, « Vaincre la suspicion, entrer dans une intimité douloureuse : une intenable extériorité ", in Bouillon F., Fresia M., et Tallio V. (éds.), Terrains sensibles. Expériences actuelles de l'anthropologie, Paris, EHESS: 55-74.

DIVAY S., 2004, "L'avortement : une déviance légale ", Déviance et Société, vol. $28: 195-209$.

ELIAS N., 1995, Engagement et distanciation: contributions à la sociologie de la connaissance, Paris, Fayard.

FASSIN D., 1999, «L'anthropologie entre engagement et distanciation. Essai de sociologie des recherches en sciences sociales sur le sida en Afrique ", in Becker C., Dozon J.P., Obbo C. et Touré M. (éds.), Vivre et penser le sida en Afrique, Paris, Codesria/Karthala/IRD : 41-66. 
GUILLAUME A., 2004, " Abortion in Africa. A review of literature from the 1990's to the present days ", CEPED, URL : http://www.ceped.org/avortement/gb/index800.html.

LOSONCZY A.-M., 2002, « De l'énigme réciproque au co-savoir et au silence. Figures de la relation ethnographique ", in Ghasarian C. (éd.), De l'ethnographie à l'anthropologie réflexive. Nouveaux terrains, nouvelles pratiques, nouveaux enjeux, Paris, Armand Colin : 91-102.

OLIVIER DE SARDAN, J.-P., 1995, «La politique du terrain. Sur la production des données en anthropologie ", consulté le 10 juin 2013, URL: http://enquete.revues.org/document263.html.

OUATTARA F., 2004, "Une étrange familiarité. Les exigences de l'anthropologie "chez soi" », Cahiers d'études africaines, 175 : 635-657.

PIRINOLI C., 2004, "L'anthropologie palestinienne entre science et politique: l'impossible neutralité du chercheur ", Anthropologie et Sociétés, 28 : 165-185.

RENAHY N., SORIGNET P.E., 2006, "L'ethnographe et ses appartenances ", in Paillé P., La méthodologie qualitative: Postures de recherche et variables de terrain, Paris, Armand Colin : 9-32.

WEBER M., 1959, Le savant et le politique, Paris, Plon.

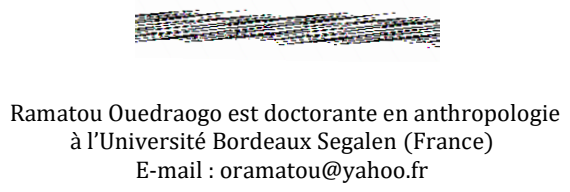

